Ophthalmologe 2017 · 114:978-985

DOI 10.1007/s00347-017-0543-6

Online publiziert: 27. Juli 2017

(c) Der/die Autor(en) 2017. Dieser Artikel ist eine Open-Access-Publikation.

CrossMark

\author{
C. Scholtyßek' $\cdot$ A. Kelber ${ }^{2}$ \\ 'The Priory Road Complex, School of Experimental Psychology, Clifton, UK \\ ${ }^{2}$ Department of Biology, Lund University, Lund, Schweden
}

\title{
Farbensehen der Tiere
}

\section{Von farbenblinden Seehunden und tetrachromatischen Vögeln}

kombinierten Signalen der Grünund Rotzapfen gegenüberstellt.

Eigenschaften beschrieben werden, wie seine Intensität, Frequenz oder den Polarisationsgrad. Farbe allerdings gehört nicht dazu. Farbe ist vielmehr eine Interpretation der spektralen Zusammensetzung des Lichtes, das von einem Objekt, das wir betrachten, reflektiert wird. Um Farbe wahrnehmen zu können, ist eine der Grundvoraussetzungen, dass die Netzhaut des Betrachters mit unterschiedlichen Zapfentypen ausgestattet ist, wobei jeder Zapfentyp für unterschiedliche Bereiche des Lichtspektrums empfindlich ist. Eine weitere Voraussetzung für Farbsehen ist, dass die Signale der verschiedenen Zapfentypen zentralnervös miteinander verglichen werden - mittels sog. Gegenfarbmechanismen.

\section{Der Mensch als Spezialfall}

Der Mensch besitzt in der Regel trichromatisches Farbensehen und 3 verschiedene Zapfentypen, die allgemein als Blau-, Grün- und Rotzapfen bezeichnet werden, da ihre maximale Empfindlichkeit in etwa den Wellenlängen des Lichtes entspricht, die wir als blau, grün oder rot wahrnehmen. Allgemein geht man davon aus, dass die Signale dieser 3 Zapfentypen in 2 primären Gegenfarbmechanismen miteinander verglichen werden:

- einem Mechanismus, der die Signale der Grünzapfen mit denen der Rotzapfen vergleicht, und

- einem weiteren Mechanismus, der die Signale der Blauzapfen den
Mit unseren 3 verschiedenen Zapfentypen ist es uns möglich, eine enorme Vielfalt an Farben zu unterscheiden. Dabei gehen die Schätzungen von läppischen 2,3 Mio. [1] bis hin zu 10 Mio. Farben [2].

Diese Vielfalt ist es wahrscheinlich, die gegen Ende des 19. Jahrhunderts, als begonnen wurde, das Farbensehen anderer Tierarten zu untersuchen, oftmals zu der irrigen Annahme führte, das Sehsystem der Tiere habe dieselbe spektrale Empfindlichkeit wie das des Menschen [3]. Heute wissen wir jedoch, dass unsere Welt der Farben eher die Ausnahme als die Regel darstellt. Im Laufe von Jahrmillionen hat die Evolution immer wieder verschiedene Varianten des Farbensehens hervorgebracht. Dabei unterscheiden sich sowohl die Anzahl der dem Farbensehen zugrunde liegenden Rezeptortypen als auch der spektrale Bereich des Lichtes, den diese Rezeptortypen abdecken. Diese Vielfältigkeit wollen wir hier anhand ausgewählter Beispiele vorstellen und erläutern.

\section{Dichromatisches Sehen: die Regel bei Säugetieren}

Was die Dimensionen des Farbensehens angeht, bilden wir Menschen innerhalb der Säugetiere eher eine Ausnahme. Die meisten Säuger sind Dichromaten [4-6]. Sie besitzen nur 2 Zapfentypen:

- einen Zapfen, dessen Empfindlichkeit im kurzwelligem Bereich des Spektrums angesiedelt ist und der unserem Blauzapfen entspricht, und
- einen weiteren Zapfen, der am empfindlichsten für langwelliges Licht ist (• Abb. 1).

Die Sehpigmente oder Opsine dieser beiden Zapfentypen werden entsprechend als SWS1 („short wavelength sensitive 1“) und LWS ("long wavelength sensitive“) bezeichnet. Während der Evolution, vor etwa 30 Mio. Jahren, ist unser Rotzapfen durch eine Verdopplung und anschließende Mutation des Gens entstanden, das bei anderen Säugetieren für das LWS-Opsin des Grünzapfens kodiert [7]. Unser Grünzapfen ist sozusagen 2-mal vorhanden, wobei die Empfindlichkeit des LWSOpsins eines dieser Zapfen zu längeren (von uns als rot wahrgenommenen) Wellenlängen hin verschoben ist.

\section{》) Dichromaten können Farbton und Sättigung nicht voneinander unterscheiden}

Dies ermöglicht es uns nicht nur, mehr Farben wahrzunehmen als die meisten anderen der Säugetiere, sondern es hat auch den Vorteil, dass wir über eine zusätzliche Dimension des Farbensehens verfügen. Wir Menschen unterscheiden grundsätzlich 3 Dimensionen von Farbe: eine achromatische Dimension, die Helligkeit, die durch die Kombination der Signale von Rot- und Grünzapfen und/ oder der Stäbchen vermittelt wird, und 2 chromatische Dimensionen: den Farbton und die Farbsättigung $[5,8]$. Wie in - Abb. 1 dargestellt, beschreibt die „Sättigung" wie stark sich ein Farbton von einem achromatischen Grau unterscheidet. Diese zusätzliche Dimension bietet 
Hier steht eine Anzeige.

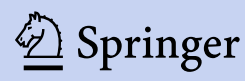




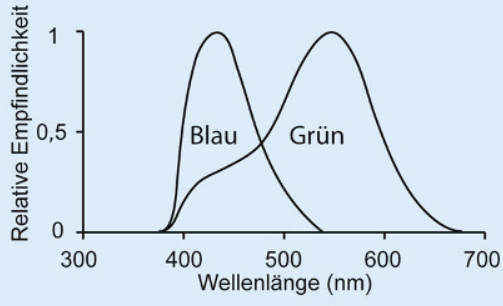

a

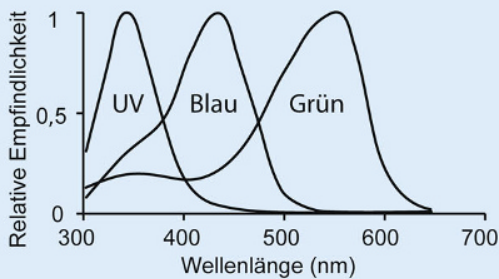

b
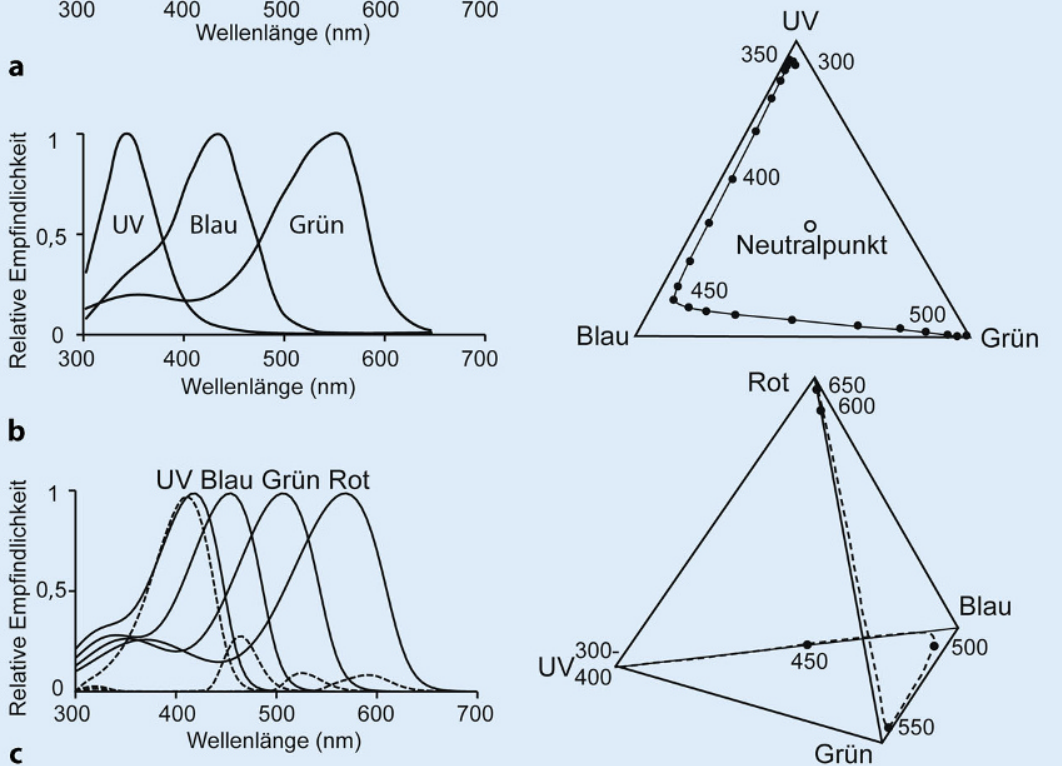

Abb. 1 ॥ Spektralempfindlichkeit der Sehzellen (links) und Chromatizitätsdiagramme (rechts) von a Pferd, b Honigbiene und c Huhn. Für das Huhn sind die Kurven ohne (durchgezogene Linien) und mit Filterung (unterbrochene Linien) durch Öltröpfchen gezeigt. In den Diagrammen rechts ist jeweils der Spektralzug mit ausgewählten Wellenlängen gezeigt. Die Eckpunkte stellen Farben dar, die nur einen Sehzellentyp erregen

einen wesentlichen Vorteil: Sie ermöglicht es uns, rein visuell Rückschlüsse auf die Materialbeschaffenheit von Objekten zu ziehen, z. B. die Stärke der Pigmentierung, Glanz oder Textur [3].

Da Dichromaten Farbton und Sättigung nicht voneinander unterscheiden können, stellt sich die Frage, wie sie Farben eigentlich wahrnehmen. Die - Abb. 1 zeigt eine schematische Darstellung des Chromatizitätsdiagramms des Pferdes. Das Farbspektrum wird hier als eindimensionales Kontinuum der Rezeptoraktivierung dargestellt. Die beiden Extreme stellen Wellenlängen bzw. Farben dar, die entweder hauptsächlich den SWS1-Zapfen oder hauptsächlich den LWS-Zapfen aktivieren. In der Mitte dieses Kontinuums, am neutralen Punkt, befinden sich Farben, die beide Rezeptortypen gleichermaßen aktivieren. Anders als bei Tri- oder Tetrachromaten können diese Farben nicht von Grau un- terschieden werden, das ebenfalls beide Zapfentypen gleichermaßen aktiviert.

Wir wissen, dass Menschen und Küken Grau qualitativ anders wahrnehmen als chromatische Farben, wie z. B. Blau, Orange oder Rot $[9,10]$. Ein Farbkontinuum, das durch den achromatischen Punkt verläuft, wird durch diesen in 2 unterschiedliche Kategorien unterteilt [9]. Bei Dichromaten stellt sich daher die Frage, ob der Neutralpunkt deren eindimensionalen Farbraum ebenfalls in $2 \mathrm{Ka}$ tegorien unterteilen [11] oder ob der Farbraum als Kontinuum wahrgenommen wird [12]. Diese Frage wurde in einer Studie von Roth, Balkenius und Kelber [13] beantwortet. Pferde wurden darauf dressiert, 2 unterschiedliche Farben, die in einiger Distanz voneinander auf dem Kontinuum lokalisiert waren, mit einer Belohnung zu assoziieren. Gleichzeitig lernten die Pferde eine weitere Farbe, die hauptsächlich einen der beiden Rezeptortypen aktivierte, nicht mit einer Beloh- nung zu assoziieren. Anschließende Tests zeigten, dass Pferde eine neue Farbe, die genau zwischen den aus der Dressur bekannten belohnten Farben liegt, ebenfalls mit einer Belohnung assoziieren. Das Interessante dabei ist, dass sie dies auch tun, wenn die neue Testfarbe genau auf dem neutralen Punkt liegt. Bei Küken wurde zuvor gezeigt, dass sie genau dies nicht tun [9]. Sie behandeln Grau als komplett anders als die mit einer Belohnung verbundenen chromatischen Farben. Die Tatsache, dass Pferde Grau genauso wie chromatische Farben behandelten, zeigt, dass der neutrale Punkt bei ihnen, anders als beim Menschen, den Farbraum nicht in 2 Kategorien unterteilt.

\section{Warum sich mit weniger begnügen?}

Wir haben bereits erwähnt, dass trichromatisches Farbensehen gegenüber dem dichromatischen Farbsehen Vorteile erbringt. Warum „begnügen“ die meisten Säuger (mit Ausnahme der Altweltaffen, Neuweltaffen, Menschenaffen und Menschen) sich dennoch mit einer Farbdimension weniger? Um diese Frage zu beantworten, müssen wir uns auf eine Zeitreise begeben. Bei den Wirbeltieren, zu denen auch die Säuger gehören, unterscheidet man 4 Genfamilien der Zapfen-Opsine. Diese sind neben den bereits erwähnten SWS1- und LWS-Genen, die RH1- und SWS2-Gene (• Abb. 2). Bei Neunaugen, vielen Fischen sowie den meisten Reptilien und Vögeln wurden alle 4 Gene gefunden. Deshalb geht man davon aus, dass die Vorfahren der heutigen Säuger, die Synapsida, ebenfalls 4 Zapfenopsine hatten und Tetrachromaten waren ([3, 14]; - Abb. 2 und 3). Während des Erdmittelalters, vor etwa 251 Mio. Jahren, koexistierten die Synapsida mit den Archosauriern, die so gut wie jede ökologische Nische dominierten. Das überlebten die Synapsiden nur, indem sie sich an eine vorwiegend nachtaktive Lebensweise anpassten. Erst am Ende der Kreidezeit, als es zum Massenaussterben der Archosaurier kam, wurden Säuger wieder tagaktiv. Die lange nachtaktive Phase der Säugetiervorfahren führte zu einer Anpassung des Auges an geringe Lichtverhältnisse [15]. Dabei spielt Farbensehen eine un- 
tergeordnete Rolle, während hohe Lichtempfindlichkeit und gutes Stäbchensehen wichtig waren, um bei Nacht hinreichend gut sehen zu können. Die Vorfahren der heutigen Säuger verloren also im Laufe der Evolution 2 der 4 ursprünglichen Vertebraten-Opsingene (• Abb. 2 und 3) und wurden zu Dichromaten, um einer hohen Anzahl weitaus lichtempfindlicherer Stäbchen Platz zu machen.

Aber auch für einige tagaktive Raubtiere und Insektenfresser kann weniger mehr sein. Eine Strategie von Beutetieren, um von Räubern schwerer entdeckt zu werden, ist es, farblich mit dem Hintergrund $\mathrm{zu}$ verschmelzen. In einer Studie von Morgan und Mollon [16] sollten menschliche Probanden - sowohl normal farbtüchtige Trichromaten als auch Dichromaten - gemusterte Objekte vor einem Hintergrund mit unterschiedlichem Muster detektieren. Dabei hatten Objekt und Hintergrund entweder dieselbe Farbe, oder beide wurden zusätzlich mit $\mathrm{Zu}$ fallsfarbmustern versehen. Für Trichromaten erschwerte diese Form der Camouflage die Detektion des Objekts deutlich, nicht aber für Dichromaten. Es ist also durchaus möglich, dass dichromatische Raubtiere kryptische Beute besser detektieren können als ihre trichromatische Konkurrenz.

\section{UV-Empfindlichkeit und Farbensehen bei Säugern}

Studien zur Evolution der VertebratenOpsine ergaben nicht nur, dass die Vorfahren der heutigen Säuger Tetrachromaten waren. Es zeigte sich auch, dass die SWS1-Opsine, die bei uns Menschen und vielen anderen tagaktiven Säugern für blaues Licht empfindlich sind, bei vielen Reptilien, Vögeln und Fischen für ultraviolettes (UV-)Licht am empfindlichsten sind ([3, 4]; • Abb. 2). Man geht also davon aus, dass unsere Blauzapfen ursprünglich UV-Zapfen waren und sich im Laufe der Evolution durch Mutationen des SWS1-Opsingens die Empfindlichkeit des SWS1-Opsins zu längeren Wellenlängen hin verschoben hat $[3,4]$.

Selbst ohne UV-Opsin könnten wir theoretisch UV-Licht sehen, denn die Empfindlichkeit aller Sehpigmente erstreckt sich bis in den ultravioletten

Ophthalmologe 2017·114:978-985 DOI 10.1007/s00347-017-0543-6

(c) Der/die Autor(en) 2017. Dieser Artikel ist eine Open-Access-Publikation.

\section{Scholtyßek · A. Kelber}

\section{Farbensehen der Tiere. Von farbenblinden Seehunden und tetrachromatischen Vögeln}

\section{Zusammenfassung}

Hintergrund. Die Farbe, in der wir einen Gegenstand sehen, ist von der spektralen Zusammensetzung des reflektierten Lichtes abhängig, stellt aber eine Interpretation unseres Auges und trichromatischen Sehsystems dar.

Fragestellung. Wie sehen Tiere anderer Arten die Welt?

Ergebnisse. Die Mehrzahl der Säugetiere hat nicht 3, sondern nur 2 Zapfentypen und daher dichromatisches Farbensehen. Marine Säuger und einige nachtaktive Säugetiere haben sogar nur 1 Zapfentypen und sind völlig farbenblind. Vögel sowie viele Fische und Reptilien dagegen sehen die Welt in mehr Farbtönen und mit 4 Zapfentypen. Viele Wirbeltiere, Insekten und Krebstiere sehen nicht nur das für uns wahrnehmbare Spektrum, sondern auch ultraviolette Strahlung als Licht.

Schlussfolgerungen. Um zu verstehen, wie Tiere anderer Arten die Welt sehen, muss man ihr Sehsystem verstehen und die Tiere in Verhaltensversuchen testen.

\section{Schlüsselwörter}

Zapfen · Netzhaut · Lichtspektrum · UVStrahlung · Dämmerungssehen

\section{Color vision in animals. From color blind seals to tetrachromatic vision in birds}

\section{Abstract}

Background. The colors in which we see an object are not only dependent on the spectral composition of the reflected light but also represent an interpretation by our eyes and the trichromatic visual system.

Objective. How do animals of other species see the world?

Results. The majority of mammals do not have three but only two types of cones and therefore have dichromatic color vision. Marine mammals and some nocturnally active mammals even have only one type of cone and are completely color blind. In contrast, birds as well as many fish and reptiles see in the world in more color hues and with four types of cones. Many vertebrates, insects and crustaceans can see not only the spectrum perceived by us but also ultraviolet radiation as light.

Conclusion. In order to understand how animals of other species see the world, their visual systems must be understood and the animals must be tested in behavioral investigations.

\section{Keywords}

Cones, retinal · Retina · Light spectrum · UV radiation - Twilight vision
Bereich. Allerdings absorbiert unsere Augenlinse UV-Licht, sodass dieses die Sehzellen gar nicht erst erreicht. Patienten, denen bei einer Kataraktoperation die Linse entfernt oder eine künstliche, UV-durchlässige Linse eingesetzt wurde, konnten anschließend tatsächlich UVLicht wahrnehmen [17-19]. Untersuchungen anderer Säugerarten zeigten, dass UV-durchlässige Linsen und daher UV-Empfindlichkeit bei Säugern sehr viel weiter verbreitet ist als ursprünglich vermutet [20]. Es gibt sogar einige Nager-, Fledermaus- und Beuteltierarten, die nicht nur UV-durchlässige Linsen haben, sondern auch ein SWS1-
Opsin, das wie bei ursprünglichen Säugern UV-empfindlich ist (s. Jacobs [4]). $\mathrm{Zu}$ diesen Arten gehören die Ratte, die Maus und der Degu. Verhaltensstudien zeigten, dass diese Arten lernen können, UV-Licht von „sichtbarem“ Licht farblich $\mathrm{zu}$ unterscheiden. Allerdings brauchte es extrem viel Zeit und Geduld, bis sie diese Farbunterscheidung lernten. Eine bahnbrechende Studie von Joesch und Meister [21] zeigte, dass das UV-Farbsehen bei Mäusen unter bestimmten Bedingungen nicht etwa auf dem Vergleich des UV-Zapfens mit dem Grünzapfen beruht, sondern auf einem Vergleich von Stäbchensignalen 


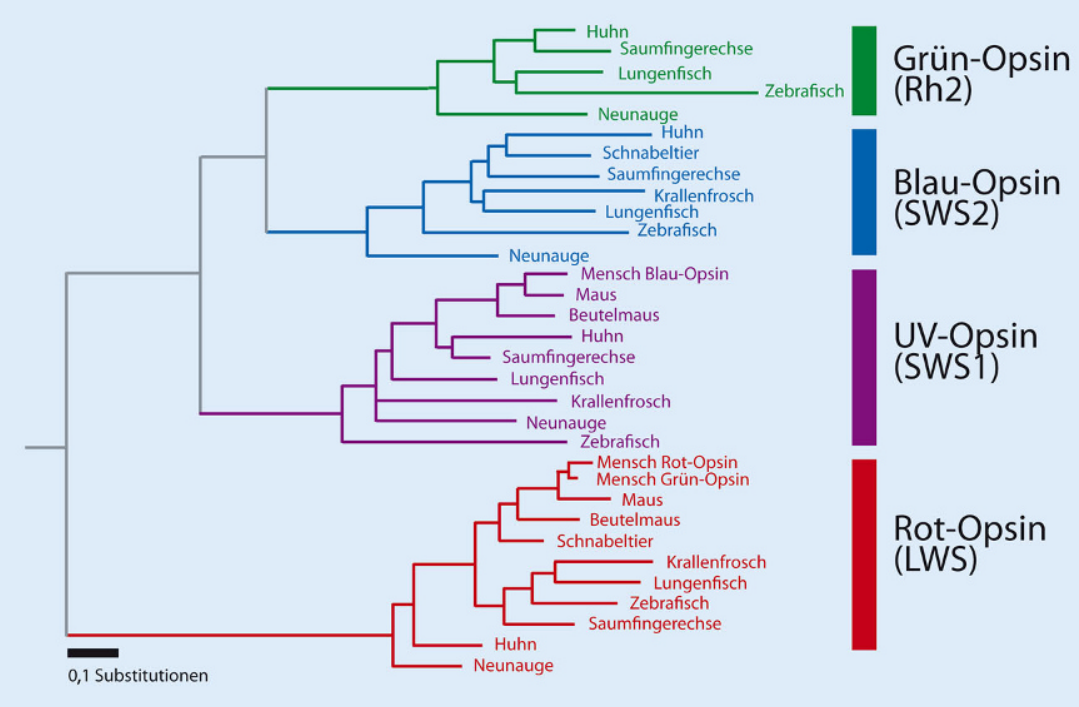

Abb. 2 ॥ Stammbaum der Zapfen-Opsingene der Wirbeltiere

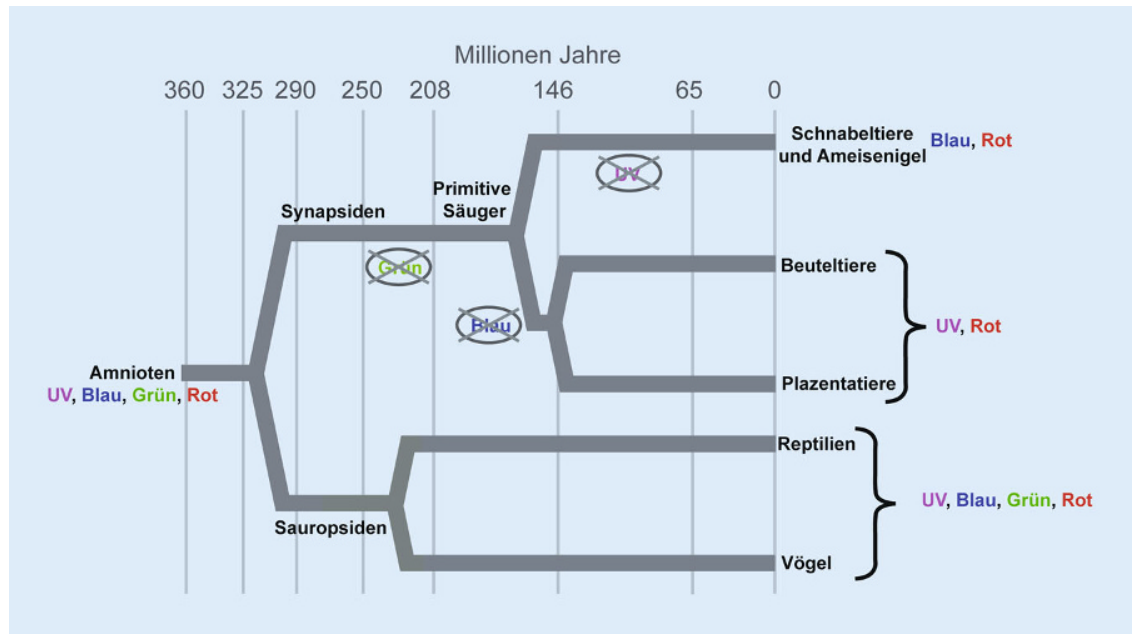

Abb. $3 \Delta$ Stammbaum der Amnioten mit Angabe der bei ihnen nachgewiesenen Zapfen-Opsine. Amnioten sind die Tetrapoden, die ihre Eier an Land legen oder im Mutterleib behalten, also Reptilien, Vögel und Säuger

mit den Signalen der UV-Zapfen. Die Autoren wiesen zudem nach, dass dieses UV-Farbensehen dazu führt, dass Mäuse Urinmarkierungen, die UV stark absorbieren, und einige Futterquellen, die UV stark reflektieren, sehr gut sehen, während sie für das menschliche Auge nur schwer auszumachen sind.

\section{Spezialfall marine Säuger}

Die Regel, dass Säugetiere 2 Zapfentypen haben, trifft auf die meisten landlebenden Arten zu. Sogar unter den nachtaktiven Säugern gibt es nur wenige Arten, die einen der beiden Zapfentypen verlo-

ren haben [22-26]. Was bei landlebenden Säugern die Ausnahme darstellt, ist bei den 2 großen Gruppen der marinen Säugetiere jedoch zur Regel geworden. Alle Wale und Robben haben im Laufe der Evolution ihre SWS1-Zapfen als Anpassung an die sekundär aquatische Lebensweise verloren [25, 27-31]. Einige Arten der Bartenwale haben sogar eine reine Stäbchenretina [32]. Trotzdem berichten einzelne Verhaltensstudien mit Seebären, Seelöwen, Seehunden oder einem Delfin, diese marinen Säuger könnten Farben sehen [33, 34], obwohl all diese Arten nur LWS-Zapfen besitzen. Diese Farbwahrnehmung soll auf dem Vergleich der Zapfensignale mit Stäbchensignalen beruhen. Bei Menschen, denen 1 oder 2 Zapfentypen fehlen (s. Jacobs [35]), bei Nachtaffen [36] und bei Mäusen [21] hat man gefunden, dass Stäbchen unter mesopischen Lichtverhältnissen tatsächlich zum Farbensehen beitragen können. Bei den Studien mit marinen Säugern kann man allerdings nicht ausschließen, dass die Versuchstiere "geschummelt" haben und die Farben in den Versuchen rein aufgrund von Helligkeitsunterschieden diskriminieren konnten [37]. Diese Zweifel an der Fähigkeit mariner Säuger, Farben sehen zu können, wurden vor Kurzem in einer Studie von Scholtyssek und Kelber untermauert [38]. Die getesteten Seehunde konnten 2 Farben unter mesopischen Verhältnissen nicht unterscheiden, wenn diese Farben für sie zweifelsfrei gleich hell waren.

Rein theoretisch ist Farbensehen für marine Säuger nicht besonders hilfreich, denn Farbensehen kompromittiert die Lichtempfindlichkeit des Auges. Und genau auf diese sind marine Säuger stark angewiesen. Wasser absorbiert und streut Licht in starkem Maße, sodass in den Tiefen, in denen marine Säuger jagen (bei Seeelefanten bis zu $1400 \mathrm{~m}$ ), nur wenig Licht vorhanden ist. Zudem werden unterschiedliche Bereiche des Spektrums unterschiedlich stark absorbiert, wodurch das Spektrum sehr schmal wird. Unter diesen Umständen ist es wahrscheinlich, dass Vorteile des Farbensehens, wie z. B. Farbkonstanz, nicht mehr bestehen. Anders als für landlebende Tiere hätte Farbensehen für marine Säuger also ausschließlich Nachteile.

\section{Meister des Farbensehens}

Nun wissen wir über farbenblinde Tiere Monochromaten - und über Dichromaten Bescheid. Das trichromatische Farbensehen ist uns bestens vertraut. Aber was bedeutet es, Tetrachromat zu sein? Diese Frage kann kein Mensch wirklich beantworten, denn bei den wenigen Menschen (ausschließlich Frauen), die diese Form des Farbensehens haben, führt sie nicht zu großen Veränderungen der Wahrnehmung, da das vierte Opsin sei- 
ne Empfindlichkeit zwischen dem Rotund dem Grün-Opsin hat [39].

Bei tetrachromatischen Fischen, Reptilien und Vögeln ist das vierte Sehpigment dagegen UV-empfindlich (• Abb. 1 und 3), und das erlaubt es diesen Tieren, die Welt tatsächlich in sehr viel mehr Farben zu sehen als wir. Mit Spektrometern und UV-empfindlichen Kameras können wir diese Farben zwar messen, aber nicht unserer eigenen Wahrnehmung zugänglich machen. Viele Fische, die im flachen Wasser der Korallenriffe leben, haben UV-Muster und können diese auch sehen [40], und die blaue Haube einer Blaumeise reflektiert ebenso viel UV- wie blaues Licht [41], die Art verdient also eigentlich den Namen UV-Meise.

I) Bei tetrachromatischen Fischen, Reptilien und Vögeln ist das vierte Sehpigment UVempfindlich

Vögel sind aber nicht nur farbenfrohe Tetrachromaten, sondern haben noch weitere Anpassungen an das Farbensehen. Jeder ihrer Zapfen ist mit einem farbigen Öltröpfchen versehen, der das Licht filtert, bevor es das Sehpigment erreicht. Das schärft die Farbunterscheidung und verbessert die Farbkonstanz [42]. Vögel können daher im für den Menschen sichtbaren Spektralbereich sehr feine Farbunterschiede sehen, wie Untersuchungen an Hühnern und Wachteln [43-45] vor Kurzem bestätigt haben. Gleichzeitig nehmen die Öltröpfchen aber auch Licht weg, sodass die absolute Empfindlichkeit der Zapfen abnimmt (• Abb. 1).

Dass Vögel zusätzlich zu Rot, Grün und Blau noch UV sehen können, muss ihre Welt wirklich farbenprächtig erscheinen lassen. Nur bei wenigen Vogelarten ist die UV-Sichtigkeit eingeschränkt, so wie bei den Greifvögeln und den Mauerseglern, deren Linse ebenso wie beim Menschen UV-Licht weitgehend absorbiert [46, 47]. Ein paar weitere Arten haben das UV-Sehen völlig verloren, darunter die Eulen, denen das UV-Opsin offenbar vollständig fehlt [48].
Hier steht eine Anzeige.

Springer 
Aber ansonsten sind Vögel die Meister des Farbensehens - jedenfalls unter den Wirbeltieren. Wenn wir dagegen das gesamte Tierreich einbeziehen, finden sich noch weitere Champions, allen voran die Schmetterlinge und die Fangschreckenkrebse. Generell ist das Farbensehen, abgesehen von den Wirbeltieren, bei Insekten und Krebsen am besten entwickelt. Diese beiden Gruppen sind nicht nur extrem artenreich, sondern auch sehr divers und haben mit ihren Facettenaugen ausgezeichnetes Sehvermögen. Wie bei allen Gliederfüßern gehört die Fähigkeit, UV-Licht zu sehen, bei ihnen zur Grundausstattung. Das wurde zuerst bei der Honigbiene entdeckt, deren von Nobelpreisträger Karl von Frisch schon vor 100 Jahren beschriebenes Farbensehen v. a. dazu dient, nektar- und pollenreiche Blüten zu entdecken (s. [5]). Während Bienen wie wir Menschen Trichromaten sind - allerdings mit einer zum UV verschobenen Spektralempfindlichkeit und mit UV-, Blau- und Grün-Opsin (- Abb. 1) - sind viele Schmetterlingsarten Tetrachromaten [49]. Das mag ihnen helfen, nicht nur Blüten zu finden, sondern auch feine Unterschiede zwischen ihren farbenprächtigen Artgenossen zu sehen und die Pflanzen für die Eiablage auszuwählen, die ihren Raupen die besten Voraussetzungen bieten. Wozu einzelne Arten allerdings 6, 7 oder bis zu 15 Sehzelltypen mit verschiedener Spektralempfindlichkeit brauchen [49], ist immer noch ein Rätsel. Und dasselbe gilt für einzelne Arten der Fangschreckenkrebse, bei denen bis zu 16 Sehzelltypen gefunden wurden [50].

\section{Farbensehen bei Nacht}

Bei Nacht sind alle Katzen grau, besagt ein altes Sprichwort, und das gilt nicht nur für uns Menschen, sondern tatsächlich für viele Tiere. Unsere Zapfen reagieren sehr schnell auf Licht, sind daher aber weniger empfindlich als unsere langsameren Stäbchen, die aber alle dieselbe Spektralempfindlichkeit haben. Daher sind wir in einer mondlosen Nacht ohne Hilfsmittel farbenblind, was weitaus besser ist, als gar nichts zu sehen. Dasselbe gilt für die meisten Wirbeltiere, die wie wir eine Duplexretina mit Stäbchen und Zapfen ha- ben. Einige Säugerarten haben, wie schon erwähnt, das Farbensehen ganz verloren. Dasselbe gilt für die Mehrzahl der Tiefseefische, die ihre Netzhaut rein mit Stäbchen bestücken [51]. Viele Vögel verlieren das Farbensehen sogar schon bei höheren Lichtintensitäten als der Mensch.

Aber es gibt Ausnahmen. Frösche und Kröten haben im Unterschied zu anderen Wirbeltieren nicht einen sondern 2 Typen Stäbchen: grünempfindliche und blauempfindliche Stäbchen. Das erlaubt es ihnen, unter bestimmten Verhältnissen auch dann Farbinformation zu verwenden, wenn alle anderen entweder gar nichts mehr sehen oder eben in Schwarz-Weiß [52]. Nachtaktive Geckos haben ähnliche Fähigkeiten, obwohl sie gar keine Stäbchen haben. Bei ihnen sind dagegen die physiologischen Eigenschaften der Zapfen an das Sehen im Dunkeln angepasst [53]. Einige Tiefseefische mit einer reinen Stäbchenretina besitzen ebenfalls 2 verschiedene Stäbchentypen mit unterschiedlicher Spektralempfindlichkeit [54]. Ob dies allerdings wie bei Fröschen und Kröten zu Farbensehen führt oder lediglich dazu beiträgt, das Kontrastsehen unter verschiedenen Lichtverhältnissen $\mathrm{zu}$ verbessern, ist bisher nicht bekannt [55].

Insekten haben keine Duplexretina, sie verwenden Tag und Nacht dieselben Sehzellen. Eine Vielzahlvon Insekten, darunter die Kakerlaken, viele Ameisen-, Grillen- und Heuschreckenarten, alle Nachtfalter, aber auch einzelne Wespenund Bienenarten sind nachtaktiv. Tatsächlich haben Versuche gezeigt, dass zumindest große Nachtfalter wie die Linien- und Weinschwärmer und nachtaktive asiatische Holzbienen auch bei Nacht die Blüten, die sie besuchen, in Farbe sehen [56].

\section{Fazit für die Praxis}

- Der Mensch verfügt über ein trichromatisches Sehsystem.

- Die Mehrzahl der Säugetiere hat nur 2 Zapfentypen und daher dichromatisches Farbensehen.

- Marine Säuger und einige nachtaktive Säugetiere haben nur 1 Zapfentypen und sind völlig farbenblind.
- Vögel sowie viele Fische und Reptilien sehen die Welt in mehr Farbtönen und mit 4 Zapfentypen.

- Viele Wirbeltiere, Insekten und Krebstiere sehen nicht nur das für uns wahrnehmbare Spektrum, sondern auch UV-Strahlung als Licht.

Korrespondenzadresse
$\begin{aligned} & \text { Prop. A. Kelber } \\ & \text { Department of Biology, Lund } \\ & \text { University } \\ & \text { Sölvegatan 35, 22362 Lund, } \\ & \text { Schweden } \\ & \text { almut.kelber@biol.lu.se }\end{aligned}$

\section{Einhaltung ethischer Richtlinien}

Interessenkonflikt. C. Scholtyßek und A. Kelber geben an, dass kein Interessenkonflikt besteht.

Dieser Beitrag beinhaltet keine von den Autoren durchgeführten Studien an Menschen oder Tieren.

Open Access. Dieser Artikel wird unter der Creative Commons Namensnennung 4.0 International Lizenz (http://creativecommons.org/licenses/by/4.0/deed. de) veröffentlicht, welche die Nutzung, Vervielfältigung, Bearbeitung, Verbreitung und Wiedergabe in jeglichem Medium und Format erlaubt, sofern Sie den/die ursprünglichen Autor(en) und die Quelle ordnungsgemäßnennen, einen Linkzur Creative Commons Lizenz beifügen und angeben, ob Änderungen vorgenommen wurden.

\section{Literatur}

1. Kleiner K (2004) What we gave up for colour vision. New Sci 181(2431):12-12

2. Wyszecki G (1969) Color. Appl Opt 8(3):602

3. Kelber A, Jacobs GH (2016) Evolution of color vision. In: Kremers J, Baraas CR, Marshall NJ (Hrsg) Human color vision. Springer, Berlin Heidelberg

4. Jacobs GH (2009) Evolution of colour vision in mammals. Philos Trans R Soc Lond, B, Biol Sci 364(1531):2957-2967

5. Kelber A, Vorobyev M, Osorio D (2003) Animal colour vision - behavioural tests and physiological concepts. Biol Rev Camb Philos Soc 78(1):81-118

6. Peichl L (2005) Diversity of mammalian photoreceptor properties: Adaptations to habitat and lifestyle? Anat Rec A Discov Mol Cell Evol Biol 287a(1):1001-1012

7. Jacobs GH, Nathans J (2009) The evolution of primate color vision. Sci Am 300(4):56-63

8. Kelber A, Osorio D (2010) From spectral information to animal colour vision: experiments and concepts. Proc Biol Sci 277(1688):1617-1625. doi:10.1098/ rspb.2009.2118

9. Jones CD, Osorio A, Baddeley RJ (2001) Colour categorization by domestic chicks. ProcRSoc Lond, B, Biol Sci 268(1481):2077-2084

10. Wyszecki G, Stiles WS (1982) Color science, 2. Aufl. Wiley, New York 
11. Jacobs GH, Deegan JF (1994) Spectral sensitivity, photopigments, and color-vision in the guinea-pig (Cavia-Porcellus). Behav Neurosci 108(5):993-1004

12. Hemmi JM (1999) Dichromatic colour vision in an Australian marsupial, the tammar wallaby. J Comp Physiol A Neuroethol Sens Neural Behav Physiol 185(6):509-515

13. Roth LSV, Balkenius A, Kelber A (2007) Colour perception in a dichromat. J Exp Biol 210(16):2795-2800

14. Jacobs $G H$ (2012) The evolution of vertebrate color vision. Adv Exp Med Biol 739:156-172

15. Walls GL (1942) The vertebrate eye and its adaptive ratiation. The Cranbrook Press, Bloomfield Hills MI

16. Morgan MJ, Mollon JD (1992) Dichromats detect colour-camouflaged objects that are not detected by Trichromats. Proc R Soc Lond, B, Biol Sci 248(1323):291-295. doi:10.1098/rspb.1992.0074

17. Anderson RM (1983) Visual perceptions and observations of an aphakic surgeon. Percept Mot Skills 57:1211-1218. doi:10.2466/pms.1983.57.3f. 1211

18. Griswold MS, Stark WS (1992) Scotopic spectral sensitivity of Phakic and Aphakic observers extending into the near ultraviolet. Vision Res 32(9):1739-1743

19. Stark WS, Wagner RH, Gillespie CM (1994) Ultraviolet sensitivity of 3 cone types in the Aphakic observer determined by chromatic adaptation. Vision Res 34(11):1457-1459

20. Douglas RH, Jeffery G (2014) The spectral transmission of ocular media suggests ultraviolet sensitivity is widespread among mammals. Soc 281(1780):20132995. doi:10.1098/rspb.2013.2995

21. Joesch M, Meister M (2016) A neuronal circuit for colour vision based on rod-cone opponency. Nature 532(7598):236. doi:10.1038/nature 17158

22. Deegan JF, Jacobs GH (1996) Spectral sensitivity and photopigments of a nocturnal prosimian, the bushbaby (Otolemur crassicaudatus). Am J Primatol 40(1):55-66

23. Jacobs GH (2013) Losses of functional opsin genes, short-wavelength cone photopigments, and color vision - A significant trend in the evolution of mammalian vision. Vis Neurosci 30(1-2):39-53

24. Jacobs GH, Neitz M, Neitz J (1996) Mutations in $\mathrm{S}$-cone pigment genes and the absence of colour vision in two species of nocturnal primate. Proc $R$ Soc Lond, B, Biol Sci 263(1371):705-710

25. Peichl L, Moutairou K (1998) Absence of shortwavelength sensitive cones in the retinae of seals (Carnivora) and African giant rats (Rodentia). Eur J Neurosci 10(8):2586-2594

26. Peichl L, Pohl B (2000) Cone types and cone/rod ratios in the crab-eating racoon and coati. Invest Ophthalmol Vis Sci 4(1):494

27. Crognale MA, Levenson DH, Ponganis PJ, Deegan JF, Jacobs GH (1998) Cone spectral sensitivity in the harbor seal (Phoca vitulina) and implications for colorvision. Can JZool 76(11):2114-2118

28. Fasick Jl, Cronin TW, Hunt DM, Robinson PR (1998) The visual pigments of the bottlenose dolphin (Tursiops truncatus). Vis Neurosci 15(4):643-651

29. Levenson DH, Dizon A (2003) Genetic evidence for the ancestral loss of SWS cone pigments in mysticete and odontocete cetaceans. Proc R Soc Lond, B, Biol Sci 270:673-679

30. Levenson DH, Ponganis PJ, Crognale MA, Deegan JF, Dizon A, Jacobs GH (2006) Visual pigments of marine carnivores: pinnipeds, polar bear, and sea otter. J Comp Physiol A 192(8):833-843
31. Newman LA, Robinson PR (2005) Cone visual pigments of aquatic mammals. Vis Neurosci 22(6):873-879

32. Meredith RW, Gatesy J, Emerling CA, York VM, Springer MS (2013) Rod monochromacy and the coevolution of cetacean retinal Opsins. Plos Genet 9(4):e1003432

33. Griebel U, Peichl L (2003) Colour vision in aquatic mammals-facts and open questions. Aquat Mamm 29(1):18-30

34. Oppermann D, Schramme J, Neumeyer C (2016) Rod-cone based color vision in seals under photopic conditions. Vision Res 124:30-40

35. Jacobs GH (1993) The distribution and nature of color-vision among the mammals. Biol Rev 68(3):413-471

36. Jacobs GH, Deegan JF, Neitz J, Crognale MA, Neitz M (1993) Photopigments and color-vision in the nocturnal monkey, Aotus. Vision Res 33(13):1773-1783

37. Scholtyssek C, Kelber A, Dehnhardt G (2008) Brightness discrimination in the harbor seal (Phoca vitulina). Vision Res 48(1):96-103

38. Scholtyssek C, Kelber A, Dehnhardt G (2015) Why do seals have cones? Behavioural evidence for colour-blindness in harbour seals. Anim Cogn 18(2):551-560

39. Jordan G, Deeb SS, Bosten JM, Mollon JD (2010) The dimensionality of color vision in carriers of anomalous trichromacy. J Vis 10(8):12

40. Siebeck UE, Parker AN, Sprenger D, Mathger LM, Wallis $G$ (2010) A species of reef fish that uses ultraviolet patterns for covert face recognition. Curr Biol 20(5):407-410

41. Andersson S, Ornborg J, Andersson M (1998) Ultraviolet sexual dimorphism and assortative mating in blue tits. Proc R Soc Lond, B, Biol Sci 265(1395):445-450

42. Vorobyev M (2003) Coloured oil droplets enhance colour discrimination. Proc Biol Sci 270(1521):1255-1261

43. Olsson P, Lind O, Kelber A (2015) Bird colour vision: behavioural thresholds reveal receptor noise. J Exp Biol 218(2):184-193

44. Olsson P, Wilby D, Kelber A (2016) Quantitative studies of animal colour constancy: using the chicken as model. Proc R Soc Lond, B, Biol Sci 283(1830):20160411

45. Lim HH, Pike TW (2016) Dietary carotenoid availability affects avian color discrimination BehavEcol 27(6):1579-1584

46. Lind O, Mitkus M, Olsson P, Kelber A (2013) Ultraviolet sensitivity and colour vision in raptor foraging. J Exp Biol 216(Pt 10):1819-1826

47. Lind O, Mitkus M, Olsson P, Kelber A (2014) Ultraviolet vision in birds: the importance of transparent eye media. Proc R Soc Lond, B, Biol Sci 281(1774):20132209

48. Wu YH, Hadly EA, Teng WJ, Hao YY, Liang W, Liu Y, Wang HT (2016) Retinal transcriptome sequencing sheds light on the adaptation to nocturnal and diurnal lifestyles in raptors. Sci Rep. doi:10.1038/ srep33578

49. Arikawa K, Stavenga DG (2014) Insect photopigments: photoreceptor spectral sensitivities and visual adaptations. In: Hunt DM, Hankins MW, Collin SP, Marshall NJ (Hrsg) Evolution of visual and non-visual pigments. Springer, New York, S 137-162

50. Cronin TW, Bok MJ, Marshall NJ, Caldwell RL (2014) Filtering and polychromatic vision in mantis shrimps: themes in visible and ultraviolet vision. Philos Trans R Soc Lond, B, Biol Sci 369(1636):20130032
51. Hirt B, Wagner HJ (2005) The organization of the inner retina in a pure-rod deep-sea fish. Brain BehavEvol 65(3):157-167

52. Yovanovich CAM, Koskela SM, Nevala N, Kondrashev SL, Kelber A, Donner K (2017) The dual rod system of amphibians supports colour discrimination at the absolute visual threshold. Philos Trans $\mathrm{R}$ Soc Lond, B, Biol Sci 372(1717):20160066

53. Roth LSV, Kelber A(2004) Nocturnal colour vision in geckos. ProcR Soc Lond, B, Biol Sci 271:485-S487

54. Hasegawa El, Sawada K, Abe K, Watanabe K, Uchikawa K, Okazaki Y, Toyama M, Douglas $\mathrm{RH}$ (2008) The visual pigments of a deep-sea myctophid fish Myctophum nitidulum Garman; an HPLC and spectroscopic description of a nonpaired rhodopsin-porphyropsin system. J Fish Biol 72(4):937-945

55. Douglas RH, Partridge JC (1997) On the visual pigments of deep-sea fish. J Fish Biol 50(1):68-85

56. Kelber A, Yovanovich C, Olsson P (2017) Thresholds and noise limitations of colour vision in $\operatorname{dim}$ light. Philos Trans R Soc Lond, B, Biol Sci 372(1717):20160065 